\title{
Linear Simplification of Dynamic Model for Printed Circuit Boards
}

\author{
Hui Wei and Liangjun $\mathrm{Xu}$ \\ Beijing University of Post and Telecommunications, Beijing, China
}

\begin{abstract}
Printed circuit board as the carrier of electronic components, the vibration characteristic of printed circuit board is related to the performance of the whole electronic equipment and the stability of the system. The printed circuit board material has non-linear characteristics, and because in engineering applications to solve the nonlinear vibration problem is very complex, and there is no suitable software to calculate. Therefore, this paper focuses on how to linearize the nonlinear vibration dynamic model of printed circuit board. In addition, the effect of concentrated mass on printed circuit board is studied by means of experimental modal analysis, which provides a simple and convenient method for studying the vibration characteristics of printed circuit boards. Circuit board on the reliability of components provided an important basis.
\end{abstract}

Keywords-printed circuit board; finite element; linear vibratio; nonlinear vibration; vibration test system; modal analysis; natural frequency; vibration mode; reliability

\section{INTRODUCTION}

Most of the various types of electronic components in electronic equipment through the through-hole installation and surface mount installation installed on the printed circuit board. While the electronic equipment in the use and transportation process, it is unavoidable to receive various forms of vibration, or even a line fault caused by vibration. The object of this paper is copper clad laminate(CCL) for printed circuit board (FR-4), the geometric dimension is $128 * 78 * 1.4 \mathrm{~mm}$, and the copper foil on the copper clad plate is completely etched. As the printed circuit board with CCL composition of epoxy resin is a viscoelastic material, so the printed circuit board material properties with viscoelastic characteristics. And the viscoelastic material of the stiffness and damping are not constant [1], but the frequency-related changes, so the printed circuit board vibration characteristics with non-linear characteristics. In the past [7 12], the vibration characteristics of printed circuit boards were considered as ordinary elastic materials, regardless of the influence of material nonlinearity on the vibration characteristics. However, with the development of the high density and miniaturization of electronic products, the accurate determination of the vibration characteristics of printed circuit boards is becoming more and more important. In this paper, the nonlinear properties of the printed circuit board are studied, and then the nonlinear vibration problem is simplified as linear vibration according to the measured parameter curve of the measured material. Then, the finite element software ANSYS is used to linearize the CCL Theoretical modal analysis, and finally using the experimental modal analysis to verify the correctness of the finite element model and to modify the finite element model.

\section{THE LiNEAR VIBRATION THEORY OF MODAL ANALYSIS}

Modal analysis [2] is an important branch of vibration theory, which is a modern method to study the dynamic characteristics of structures. It is the application of system identification method in engineering vibration field. According to the method and method of modal analysis, modal analysis can be divided into theoretical modal analysis and experimental modal analysis. The theory of modal analysis is based on the linear vibration theory, study motivation, system response, the relationship between the three; experimental modal analysis is the inverse process of the theory of modal analysis, through measuring the time course of excitation and response, by using digital signal processing technique to obtain the frequency response function or impulse response function, the nonparametric model of the system is obtained, and then the modal parameters of the system are obtained by parameter identification method and the physical parameters of the system are further determined.

Mechanical vibration systems are usually composed of quality, stiffness and damping elements. As the printed circuit board is always equipped with electronic components, and printed circuit board itself is a distribution of quality, stiffness and damping distribution system. So the printed circuit board is often simplified to include a number of concentrated mass, concentrated stiffness and damping of the multi-degree of freedom vibration system.

The general expression of the differential equation of motion with $\mathrm{n}$ degrees of freedom vibration system is $[3 \sim 5]$ :

$$
\begin{aligned}
& {\left[\begin{array}{cccc}
m_{11} & m_{12} & \cdots & m_{1 n} \\
m_{21} & m_{22} & \cdots & m_{2 n} \\
\vdots & \vdots & & \vdots \\
m_{n 1} & m_{n 2} & \cdots & m_{n n}
\end{array}\right]\left\{\begin{array}{c}
\ddot{z}_{1} \\
\ddot{z}_{2} \\
\vdots \\
\ddot{z}_{1}
\end{array}\right\}+\left[\begin{array}{cccc}
c_{11} & c_{12} & \cdots & c_{1 n} \\
c_{21} & c_{22} & \cdots & c_{2 n} \\
\vdots & \vdots & & \vdots \\
c_{n 1} & c_{n 2} & \cdots & c_{n n}
\end{array}\right]\left\{\begin{array}{c}
\dot{z}_{1} \\
\dot{z}_{2} \\
\vdots \\
\dot{z}_{n}
\end{array}\right\}} \\
& +\left[\begin{array}{cccc}
k_{11} & k_{12} & \cdots & k_{1 n} \\
k_{21} & k_{22} & \cdots & k_{2 n} \\
\vdots & \vdots & & \vdots \\
k_{n 1} & k_{n} & \cdots & k_{n n}
\end{array}\right]\left[\begin{array}{c}
z_{1} \\
z_{2} \\
\vdots \\
z_{n}
\end{array}\right]=\left\{\begin{array}{c}
q_{1}(t) \\
q_{2}(t) \\
\vdots \\
a_{n}(t)
\end{array}\right\}
\end{aligned}
$$
form:

The above formula can be written in the following simpler

$$
[M]\{\ddot{z}\}+[C]\{\dot{z}\}+[K]\{z\}=\{q(t)\}
$$


Where $[\mathrm{M}],[\mathrm{C}],[\mathrm{K}]$ are called mass, damping and stiffness matrices, respectively, $\mathrm{n} \times \mathrm{n}$ square. $\{\ddot{z}\},\{\dot{z}\},\{z\},\{q(t)\}$ are called acceleration, velocity and displacement vector column matrices, and excitation force vector arrays, respectively. The process of modal analysis is to solve the above differential equations, and obtain the modal parameters of the vibration system, such as the natural frequencies and the main modes. The solution process is as follows.

First, the damping matrix [C] and the exciting force array $\{\mathrm{q}(\mathrm{t})\}$ in the above equation are zero matrix, and the general form of the no damping free vibration differential equation of $n$ degree of freedom system is obtained as follows:

$$
\left[\begin{array}{cccc}
m_{11} & m_{12} & \cdots & m_{1 n} \\
m_{21} & m_{22} & \cdots & m_{2 n} \\
\vdots & \vdots & & \vdots \\
m_{n 1} & m_{n 2} & \cdots & m_{n n}
\end{array}\right]\left\{\begin{array}{c}
\ddot{z}_{1} \\
\ddot{z}_{2} \\
\vdots \\
\ddot{z}
\end{array}\right\}+\left[\begin{array}{cccc}
k_{11} & k_{12} & \cdots & k_{1 n} \\
k_{21} & k_{22} & \cdots & k_{2 n} \\
\vdots & \vdots & & \vdots \\
k_{n 1} & k_{n 2} & \cdots & k_{n n}
\end{array}\right]\left[\begin{array}{c}
z_{1} \\
z_{2} \\
\vdots \\
z_{n}
\end{array}\right]=\left\{\begin{array}{c}
0 \\
0 \\
\vdots \\
0
\end{array}\right\}
$$

Where $\mathrm{m}_{\mathrm{ij}}=\mathrm{m}_{\mathrm{ji}}, \mathrm{k}_{\mathrm{ij}}=\mathrm{k}_{\mathrm{ji}}$, the above formula may be abbreviated as follows:

$$
[M]\{\ddot{z}\}+[K]\{z\}=\{0\}
$$

Set up the solution as

$$
\{z\}=\{A\} \sin (\omega t+\varphi)
$$

Assuming that the system is free vibration, the displacement $Z_{i}$ of each particle is a special solution of the harmonic vibration at the same frequency $\omega_{\text {and initial angle } \varphi} \varphi$. Substituting the above equation and its second derivative into the system of equations expressed in matrix for

$$
\left([K]-\omega^{2}[M]\right)\{A\}=\{0\}
$$

$$
\text { Let }[B]=[K]-\omega^{2}[M]
$$

Where $\{A\}=\left[A_{1}, A_{2} \cdots A_{n}\right]^{T}$ is the free vibration amplitude vector array, and $[\mathrm{B}]$ is the feature matrix. For the free vibration of the system, the amplitude can not be all zero, that is, $\{A\} \neq 0$, the nonzero solution is required to make the determinant of the feature matrix zero. So there is

$$
|B|=\left|\begin{array}{cccc}
k_{11}-m_{11} \omega^{2} & k_{12}-m_{12} \omega^{2} & \cdots & k_{1 n}-m_{1 n} \omega^{2} \\
k_{21}-m_{21} \omega^{2} & k_{22}-m_{22} \omega^{2} & \cdots & k_{2 n}-m_{2 n} \omega^{2} \\
\vdots & \vdots & & \vdots \\
k_{n 1}-m_{n 1} \omega^{2} & k_{n 2}-m_{n 2} \omega^{2} & \cdots & k_{n n}-m_{n n} \omega^{2}
\end{array}\right|=0
$$

The above equation is called the characteristic equation or the frequency equation. The polynomial can get n roots: $\omega_{1}^{2}$, $\omega_{2}^{2}, \cdots, \omega_{n}^{2}$. The $\mathrm{n}$ roots are called eigenvalues, and when they are opened, we can get $\mathrm{n}$ natural frequencies arranged in descending order: $\omega_{1}<\omega_{2}<\cdots<\omega_{n}$. They are respectively called the first-order natural frequency, second-order natural frequency n-order natural frequency.

Substituting any of the eigenvalues $\omega_{i}$ into the above equation yields

$$
\left([K]-\omega_{i}^{2}[M]\right)\left\{A^{(i)}\right\}=\{0\}
$$

The amplitude vector $\left\{A^{(i)}\right\}$, called the characteristic vector, is also called the main mode or the main mode. It represents a certain form of vibration and displacement of the system, so it is also called the natural mode.

\section{Research ON MAterial Properties OF PRINTED CIRCUIT BOARD PREPARE}

The printed circuit board is composed of epoxy resin and glass fiber cloth. The epoxy resin determines the characteristics of the viscoelastic material of the printed circuit board, and the weaving structure of the glass fiber cloth determines whether the material characteristic of the CCL is anisotropic or homosexual.

In order to determine the structure of the FR-4 CCL glass fiber cloth, the FR-4 sample, which was cut into a square shape, was dissolved by using an epoxy resin sizing agent, and after several hours of dissolution, it was found that the epoxy resin was dissolved by the gumming agent, And the glass fiber is not dissolved, and then take the optical microscope to observe the appearance of fiberglass cloth shown in Figure 1, from the figure can be very clear to see the glass fiber weaving in the form of plain weave.

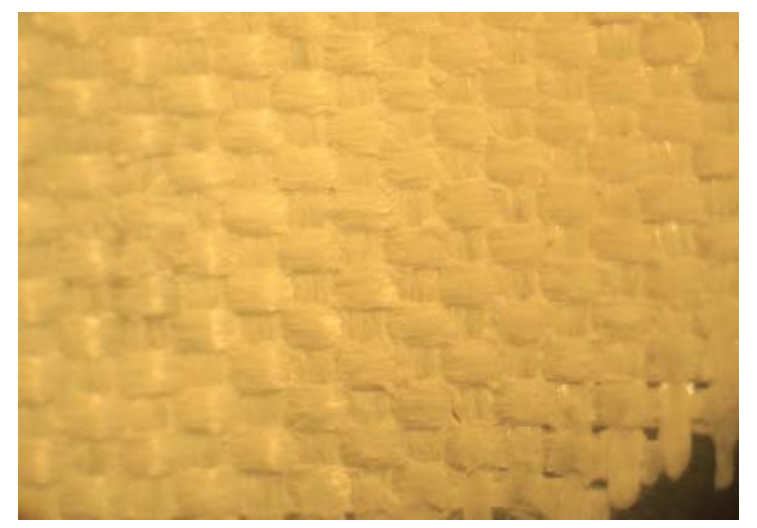

FIGURE I. FR-4 FIBERGLASS CLOTH WEAVING STRUCTURE

It can be seen, FR-4 CCL material properties of anisotropy, but also because of the fiber in which they are arranged in parallel and parallel, so the material properties of orthotropic. In ANSYS, the orthotropic material requires 9 input material parameters: $E_{1}, E_{2}, E_{3}, v_{12}, v_{13}, v_{23}, G_{12}, G_{13}, G_{23}$. Because the thickness of FR-4 CCL is $1.4 \mathrm{~mm}$, compared with the length and width, the size of the copper clad laminate is very small, so the stress and strain relation of FR-4 CCL can be approximately plane stress, Therefore, the values of $E_{3}, v_{13}$ and $v_{23}$ do not affect the calculation results. And $G_{13}, G_{23}$ value as long as the order of magnitude is correct, the calculation results 
are not affected, so need to measure the material parameters $E_{1}$, $E_{2}, v_{12}, G_{12}$.

Since the elastic modulus of the printed circuit board changes with frequency, it is necessary to measure the elastic modulus at different frequencies. First, measure the natural frequency of the different length of the sample in the cantilever mode, and then pass the following Formula calculated:

$$
E=\left\lceil\frac{4 \pi(3 \rho)^{\frac{1}{2}} l^{2}}{h}\right]^{2}\left(f_{i} / k_{i}^{2}\right)^{2}
$$

Where,

E -elastic modulus, $\mathrm{Pa}$; $\rho_{\text {- }}$ material density, $\mathrm{Kg} / \mathrm{m}^{3}, 1$ - the free length of the specimen in the cantilever mode, $\mathrm{m} ; \mathrm{H}$ thickness, $\mathrm{m}$; i- resonance order; fi -the i order resonance frequency, $\mathrm{Hz} ; k_{i}^{2}$ The numerical calculation factor at the ith order resonance, which is determined by the following equations

Cantilever beam mode:

$$
\begin{aligned}
& \mathrm{k}_{\mathrm{i}}^{2}=3.52(\mathrm{i}=1) \\
& \mathrm{k}_{\mathrm{i}}^{2}=3.52(\mathrm{i}=1) \\
& \mathrm{k}_{\mathrm{i}}^{2}=22.0(\mathrm{i}=2) \\
& \mathrm{k}_{\mathrm{i}}^{2}=(\mathrm{i}-0.5)^{2} \pi^{2}(\mathrm{i}>2)
\end{aligned}
$$

$E_{1}$ is obtained by measuring the natural frequency of samples of different lengths in the length of FR-4 CCL. $E_{2}$ is obtained by measuring the natural frequencies of different lengths of FR-4 CCL widths. $G_{12}$ is obtained by length, width, and elasticity in 45 degrees Modulus and Poisson's ratio $v_{21}$. Length, width and 45 degrees direction of the elastic modulus of the measurement results shown in Table 1 to 3.

TABLE I. THE ELASTIC MODULUS OF FR-4 CCL IN LENGTH DIRECTION

\begin{tabular}{|l|l|l|l|l|}
\hline $\begin{array}{c}\text { Length } \\
(\mathbf{m m})\end{array}$ & $\begin{array}{c}\text { Width } \\
(\mathbf{m m})\end{array}$ & $\begin{array}{c}\text { Thickness } \\
(\mathbf{m m})\end{array}$ & $\begin{array}{c}\text { natural } \\
\text { frequency } \\
\mathbf{( H z )}\end{array}$ & $\begin{array}{c}\mathbf{E}_{\mathbf{1}} \\
(\mathbf{e 1 0 P a})\end{array}$ \\
\hline 238 & 10 & 14.4 & 14.4 & 2.75 \\
\hline 170 & 10 & 14.4 & 27.8 & 2.67 \\
\hline 130 & 10 & 14.4 & 47.0 & 2.61 \\
\hline 110 & 10 & 14.4 & 65.0 & 2.56 \\
\hline 90 & 10 & 14.4 & 96.0 & 2.50 \\
\hline 70 & 10 & 14.4 & 155.3 & 2.39 \\
\hline 50 & 10 & 14.4 & 294.6 & 2.24 \\
\hline
\end{tabular}

TABLE II. THE ELASTIC MODULUS OF FR-4 CCL IN WIDTH DIRECTION

\begin{tabular}{|l|l|l|l|l|}
\hline $\begin{array}{c}\text { Length } \\
(\mathbf{m m})\end{array}$ & $\begin{array}{c}\text { Width } \\
\mathbf{( m m )}\end{array}$ & $\begin{array}{c}\text { Thickness } \\
\mathbf{( m m )}\end{array}$ & $\begin{array}{c}\text { natural } \\
\text { frequency } \\
\mathbf{( H z )}\end{array}$ & $\begin{array}{c}\mathbf{E}_{\mathbf{1}} \\
(\mathbf{e 1 0 P a})\end{array}$ \\
\hline 240 & 10 & 1.4 & 14.8 & 3.00 \\
\hline 170 & 10 & 1.4 & 28.7 & 2.84 \\
\hline 150 & 10 & 1.4 & 36.9 & 2.85 \\
\hline 130 & 10 & 1.4 & 48.9 & 2.82 \\
\hline 110 & 10 & 1.4 & 67.7 & 2.77 \\
\hline 90 & 10 & 1.4 & 99.5 & 2.68 \\
\hline 80 & 10 & 1.4 & 125.3 & 2.65 \\
\hline 70 & 10 & 1.4 & 160.7 & 2.56 \\
\hline 60 & 10 & 1.4 & 217.0 & 2.52 \\
\hline 50 & 10 & 1.4 & 306.5 & 2.42 \\
\hline
\end{tabular}

TABLE III. THE ELASTIC MODULUS OF FR-4 CCL IN 45 DEGREE DIRECTION

\begin{tabular}{|l|l|l|l|l|}
\hline $\begin{array}{c}\text { Length } \\
(\mathbf{m m})\end{array}$ & $\begin{array}{c}\text { Width } \\
\mathbf{( m m})\end{array}$ & $\begin{array}{c}\text { Thickness } \\
\mathbf{( m m )}\end{array}$ & $\begin{array}{c}\text { natural } \\
\text { frequency } \\
\mathbf{( H z )}\end{array}$ & $\begin{array}{c}\mathbf{E}_{\mathbf{1}} \\
(\mathbf{e 1 0 P a})\end{array}$ \\
\hline 240 & 10 & 1.4 & 11.8 & 1.84 \\
\hline 170 & 10 & 1.4 & 23.2 & 1.91 \\
\hline 130 & 10 & 1.4 & 39.6 & 1.86 \\
\hline 110 & 10 & 1.4 & 54.6 & 2.03 \\
\hline 90 & 10 & 1.4 & 80.8 & 2.08 \\
\hline 70 & 10 & 1.4 & 131.2 & 2.10 \\
\hline 60 & 10 & 1.4 & 177.2 & 2.06 \\
\hline 50 & 10 & 1.4 & 249.5 & 2.31 \\
\hline
\end{tabular}

The relationship between elastic modulus and frequency in each direction is obtained by curve fitting. The relationship between elastic modulus and frequency is shown in Fig. 2, and the relation formulas are as follows:

Length direction:

$E_{1}=-304.505 \mathrm{x}^{\wedge} 3+1.884 \mathrm{e} 5 \mathrm{x}^{\wedge} 2-4.822 \mathrm{e} 7 \mathrm{x}+2.804 \mathrm{e} 10$

Width direction:

$$
E_{2}=-2.9391 \mathrm{e} 2 \mathrm{x}^{\wedge} 3+1.9154 \mathrm{e} 5 \mathrm{x}^{\wedge} 2-5.0931 \mathrm{e} 7 \mathrm{x}+3.02564 \mathrm{e} 10
$$

45-degree direction:

$E_{45}=-3.157 \mathrm{e} 2 \mathrm{x}^{\wedge} 3+1.508 \mathrm{e} 5 \mathrm{x}^{\wedge} 2-3.156 \mathrm{e} 7 \mathrm{x}+1.940 \mathrm{e} 10(13)$

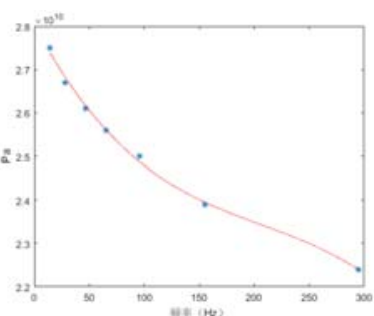

(a)Length direction

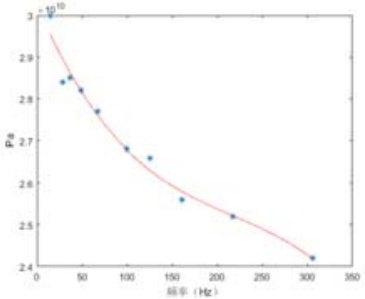

(b) Width direction 


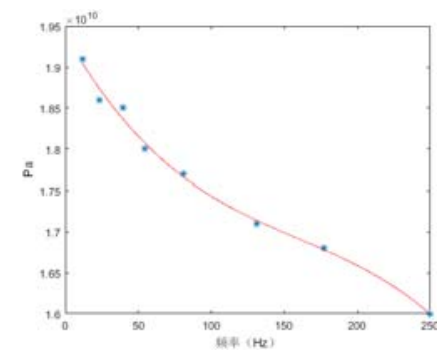

(c) $45^{\circ}$ direction

FIGURE II. RELATIONSHIP BETWEEN ELASTIC MODULUS AND FREQUENCY

It can be seen that the elastic modulus of the FR-4 CCL decreases with the increase of the frequency. Therefore, when the finite element model is established, the input elastic modulus varies with the natural frequency of the CCL different. The shear modulus is calculated as [6]:

$$
G_{12}=\frac{1}{\frac{4}{E_{45}}-\frac{1}{E_{1}}-\frac{1}{E_{2}}+\frac{2 v_{21}}{E_{1}}}
$$

The density of the material uses a balance to measure the mass of the sample of a certain size and then uses the formula to calculate.

$$
\rho=\frac{m}{a b t}
$$

Where $\mathrm{m}$ is the mass, $\mathrm{a}$ is the length, $\mathrm{b}$ is the width, $\mathrm{t}$ is the thickness, and the density of the FR-4 CCL to be studied is $2120 \mathrm{Kg} / \mathrm{m} 3$.

\section{Establish the Dynamic Model of Printed Circuit BOARD}

Finite element modal analysis is a theoretical modal analysis. Its essence is to solve the eigenvalue problem of free vibration system, that is, to solve the eigenvalue and eigenvector of the characteristic equation. Generally, the effect of damping on the natural frequency and mode of the modal parameters is very small. Therefore, the finite element modal analysis can approximate the damping to zero, which can be used to solve the eigenvalue problem of the damped free vibration system. It is also possible to consider the damping and perform the eigenvalue solution of the damped free vibration system. The basic steps of finite element modal analysis are: modeling - loading and solving - extended modal observations and post processing. In the modeling, we need to establish the geometric model of the structure, enter the appropriate material parameters (elastic modulus, Poisson's ratio and density), and select the appropriate finite element unit for the structure of the grid. Establish a geometric model and then define the boundary conditions of the structure, and finally choose the solution method. Since damping has little effect on the results of the modal analysis, the damping of the material is ignored.

When the finite element model of FR-4 CCL is established, it is necessary to determine the parameter value of the material. According to the results of the previous section, the elastic modulus of the CCL decreases with the increase of the frequency, and the relationship curve is nonlinear. As the finite element software ANSYS in the modal analysis only consider the linear, while ignoring the non-linear. In order to use ANSYS software for printed circuit board modal analysis, and in order to make the calculation results as close as possible, the selection of the material parameters of the printed circuit board shall be selected according to the actual ambient vibration frequency or the natural frequency of the printed circuit board itself. Also, when the ambient vibration frequency is close to the natural frequency of the printed circuit board, the response of the printed circuit board is the largest, so the material parameter is determined according to the natural frequency of the printed circuit board itself. In this section, it is assumed that the natural frequency of the printed circuit board is $50 \mathrm{~Hz}$, and the elastic modulus values in the length, width and direction of 45 degrees are calculated according to the equations (11 to 13). The value of $v_{12}$ is assumed to be 0.26 , and then calculates the value of the shear modulus by Equation (14). Because of the elastic modulus of the E-grade glass fiber constituting the epoxy glass fiber cloth plate is $74 \mathrm{Gpa}[6]$, the elastic modulus of the epoxy resin is $3.2 \mathrm{GPa}$. According to the composite micromechanics [6], it can be assumed: $E_{3}=3.2 \mathrm{GPa}$, $v_{13}=v_{23}=0.3, G_{13}=G_{23}=3.9 \mathrm{GPa}$. The values of the material parameters are shown in Table 4 .

TABLE IV. HYPOTHETICAL VALUES OF MATERIAL PARAMETERS FOR FR-4 CCL

\begin{tabular}{|c|c|c|c|c|c|c|c|c|}
\hline $\boldsymbol{E}_{1}$ & $\boldsymbol{E}_{\mathbf{2}}$ & $\boldsymbol{E}_{\mathbf{4 5}}$ & $\boldsymbol{v}_{\mathbf{1 2}}$ & $\boldsymbol{v}_{\mathbf{2}}$ & $\boldsymbol{v}_{\mathbf{1 3}}$ & $\boldsymbol{G}_{\mathbf{1 2}}$ & $\boldsymbol{G}_{\mathbf{2} 3}$ & $\boldsymbol{G}_{\mathbf{1 3}}$ \\
\hline $26 \mathrm{GPa}$ & $28 \mathrm{GPa}$ & $39 \mathrm{GPa}$ & 0.26 & 0.3 & 0.3 & $6.06 \mathrm{GPa}$ & $3.9 \mathrm{GPa}$ & $3.9 \mathrm{GPa}$ \\
\hline
\end{tabular}

FR-4 CCL boundary conditions for the four corners fixed, the unit type using SHELL181, divided grid after the geometric model shown in Figure 3. Then, the modal analysis is carried out to calculate the first five natural frequencies of the FR-4 CCL. See Table 5, and the first order vibration mode is shown in figure 4.

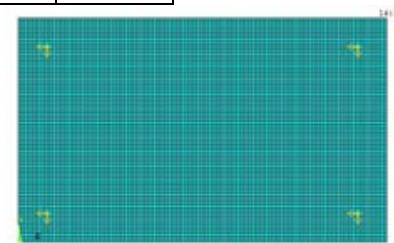

FIGURE III. GRID DIVISION AND BOUNDARY CONDITION 


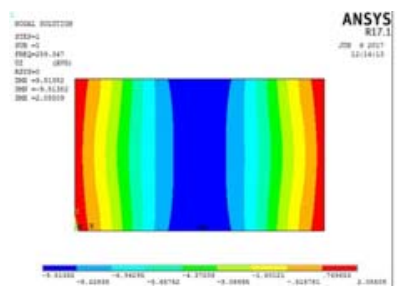

FIGURE IV. THE FIRST ORDER MODE BEFORE CORRECTION

TABLE V. FIRST FOURTH ORDER NATURAL FREQUENCY OF FR-4 CCL (BEFORE CORRECTION)

\begin{tabular}{|c|c|c|c|}
\hline First order & Second order & Third order & Fourth order \\
\hline $259.35 \mathrm{~Hz}$ & $418.68 \mathrm{~Hz}$ & $789.89 \mathrm{~Hz}$ & $888.26 \mathrm{~Hz}$ \\
\hline
\end{tabular}

\section{MOdificAtion OF Printed CIRCUIT BOARD DYNAMIC MODEL}

In order to verify the correctness of FR-4 CCL finite element model, this section designed the vibration mode experiment. The excitation system consists of signal generator, power amplifier and electric vibration table. The excitation method is to use the electric vibration exciter to carry out the basic excitation, and the signal of the vibration exciter is generated by the signal generator to produce a sinusoidal signal in a certain frequency range, and then amplified by the power amplifier to excite the exciter. The pick-up system uses an accelerometer to measure the input excitation signal of the oscillating table plane. The eddy current sensor measures the output response signal of the CCL plane and then uses the input and output signals for transfer function analysis. The peak frequency of the transfer function curve is the natural frequency of the CCL. The boundary conditions are the same as those of the finite element model, and the printed circuit board is fixedly connected with the table of the exciter with four pairs of screw studs.

The flow of the experimental modal analysis is as follows: first determine the natural frequency of copper clad plate, then calculate and calculate the vibration mode of CCL. Methods to determine the natural frequency of the sinusoidal signal is a certain frequency range by signal generator, setting the sweep speed appropriate, using the sensor to measure a time-domain signal excitation and response, and then digital signal processing, calculates the transfer function, to determine the natural frequency of CCL. The determination method of the natural frequency is to use the signal generator to generate a certain frequency range of the sinusoidal signal, and set the appropriate sweep speed, and then use the sensor to measure the excitation and response time domain signal, finally through the digital signal processing to calculate the transfer function. And determine the natural frequency of CCL. The method of determining the mode is to use the signal generator to produce a sinusoidal signal with a frequency equal to the natural frequency of the CCL. At this time, the CCL will resonate. And then by moving the position of the eddy current sensor to measure the response of a certain number of nodes on the CCL. Finally, all the response signals are analyzed by using the modal analysis software to obtain the vibration mode of the CCL. Since the response at the low frequency is greatest and the reliability of the electronic components on the printed circuit board is greatest, only the first-order mode of the CCL is tested. The first-order natural frequency of the CCL and the first-order mode are shown in Figures 5-6.

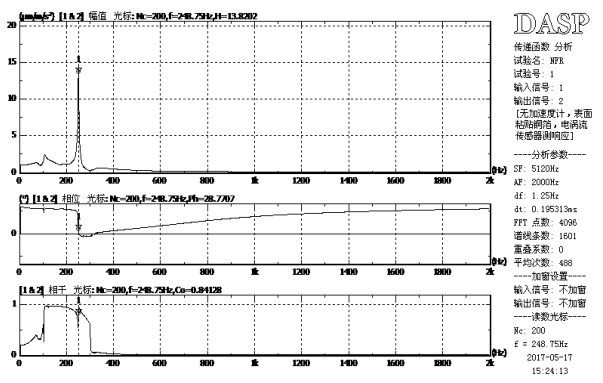

FIGURE V. FIRST-ORDER NATURAL FREQUENCY

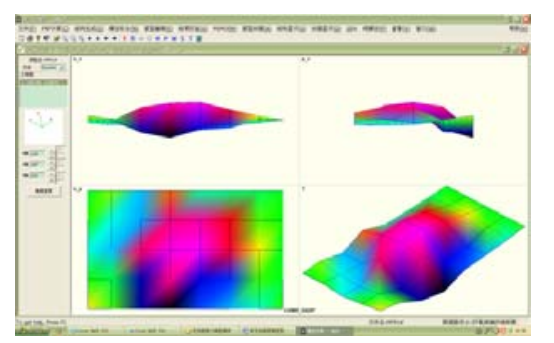

FIGURE VI. FIRST-ORDER MODE (EXPERIMENTAL)

The first-order natural frequency of FR-4 CCL is $249 \mathrm{~Hz}$, and the frequency calculated by finite element software is 259 Hz. In order to make the finite element model more realistic, the material in the model Parameters were properly modified. Since the natural frequency is $249 \mathrm{~Hz}$, the value of the elastic modulus is calculated at $249 \mathrm{~Hz}$ and the Poisson's ratio $v_{12}$ is appropriately modified. The corrected material parameters are shown in Table 6.

TABLE VI. FR-4 CCL MATERIAL PARAMETERS (AFTER CORRECTION)

\begin{tabular}{|c|c|c|c|c|c|c|c|c|}
\hline $\boldsymbol{E}_{1}$ & $\boldsymbol{E}_{\mathbf{2}}$ & $\boldsymbol{E}_{45}$ & $\boldsymbol{v}_{12}$ & $\boldsymbol{v}_{\mathbf{2}}$ & $\boldsymbol{v}_{\mathbf{1 3}}$ & $\boldsymbol{G}_{12}$ & $\boldsymbol{G}_{\mathbf{2}}$ & $\boldsymbol{G}_{\mathbf{1 3}}$ \\
\hline $23 \mathrm{GPa}$ & $25 \mathrm{GPa}$ & $39 \mathrm{GPa}$ & 0.36 & 0.3 & 0.3 & $5.12 \mathrm{GPa}$ & $3.9 \mathrm{GPa}$ & $3.9 \mathrm{GPa}$ \\
\hline
\end{tabular}

VI. The INFLUENCE OF ELECTRONIC COMPONENTS ON THE NATURAL FREQUENCY OF PRINTED CIRCUIT BOARD

Electronic components such as integrated circuits, resistors, capacitors and diodes are mounted on printed circuit boards via electrical leads. Some electronic components even greater than the weight of the printed circuit board itself. It can be speculated that the vibration characteristics of the printed circuit board on which the electronic components are mounted are different from the vibration characteristics of the printed circuit board on which the electrical components are not mounted.

In order to study the influence of electronic components on the natural frequency of printed circuit boards experimentally, 
this section uses an accelerometer to measure the response of CCL plates to simulate it. Since the mass of the accelerometer is $16 \mathrm{~g}$ and the weight of the CCL itself is only $29.6 \mathrm{~g}$, the weight of the accelerometer is bound to have an effect on the modal parameters of the CCL. In the experiment, the accelerometer is pasted on the surface of copper clad plate with 502 glue. The signal generator generates slow sweep sine signal, and the sweep speed is $0.5 \mathrm{~Hz} / \mathrm{s}$. When the accelerometer is located at different positions, the first natural frequency of the FR-4 CCL is shown in table 8.

TABLE VII. THE FREQUENCY OF THE PRINTED CIRCUIT BOARD WHEN THE ACCELEROMETER IS LOCATED AT A DIFFERENT LOCATION

\begin{tabular}{|c|l|l|l|l|l|l|l|l|l|}
\hline Acceleration position No & 2 & 3 & 4 & 10 & 11 & 12 & 18 & 19 & 20 \\
\hline first order natural frequency /Hz & 214 & 171 & 164 & 216 & 194 & 178 & 218 & 204 & 186 \\
\hline
\end{tabular}

\section{CONCLUSION}

In this paper, the theoretical modal analysis and experimental modal analysis of printed circuit boards are carried out, and the natural frequencies of printed circuit boards at different locations are tested by experiments. The following conclusions are obtained:

1) The properties of the printed circuit board are orthotropic, and the elastic modulus and the shear modulus in the length and width directions are not constant and vary with the frequency. Therefore, the printed circuit board The vibration characteristic is nonlinear vibration.

2) Since the nonlinear vibration differential equation is complicated, and the modal analysis of the finite element software can only be linearly analyzed. In order to facilitate the engineering application, the nonlinear vibration can be simplified linearly, but it needs to be simplified according to the characteristics of the printed circuit board itself. In this paper, according to the natural frequency of the printed circuit board to select the appropriate material parameter value, so that the finite element dynamic model closer to the actual situation closer to the actual situation.

3) By changing the position of the accelerometer to simulate the actual printed circuit board mounted components on the printed circuit board modal parameters, it can be drawn that the printed circuit board components are inevitably reduced the natural frequency of the printed circuit board, and the degree of reduction of different positions is not the same. So the layout of components should be made as much as possible to reduce the inherent frequency of printed circuit board and then to reduce the anti-vibration performance of printed circuit boards.

\section{REFERENCES}

[1] Chang Guanjun. "Viscoelastic damping material" [M]. National Defense Industry Press, 2012

[2] Zhang Li, Lin Jianlong, Xiang Huiyu. "Modal analysis and experiment" [M]. Tsinghua University Press, 2011.

[3] Liu Yanzhu, Chen Wenliang, Chen Liqun. Vibration dynamics. Second Edition [M]. Higher Education Press, 2011.

[4] Cyril M.Harris, Allan G.Piersol. Shock and Vibration Manual [M]. China Petrochemical Press, 2008.

[5] Zhao Zhanshu. An vibration analysis and experiment of electronic equipment [M]. Southeast University Press, 1991

[6] Shen Guanlin, Hu Gengkai, Liu Bin. Composites Mechanics. Second Edition [M]. Tsinghua University Press, 2013.

[7] Chen Y S, Wang C S, Yang Y J. Combining vibration test with finite element analysis for the fatigue life estimation of PBGA components[J]. Microelectronics Reliability, 2008, 48(4):638-644.

[8] Tong Y T, Ng H S, Lim C T, et al. Impact life prediction modeling of TFBGA packages under board level drop test[J]. Microelectronics Reliability, 2004, 44(7):1131-1142.

[9] Cifuentes A O. Estimating the dynamic behavior of printed circuit boards [J]. Components Packaging \& Manufacturing Technology Part B Advanced Packaging IEEE Transactions on, 1994, 17(1):69-75.

[10] Lee Y C, Wang B T, Lai Y S, et al. Finite element model verification for packaged printed circuit board by experimental modal analysis[J]. Microelectronics Reliability, 2008, 48(11):1837-1846.

[11] Pitarresi J, Roggeman B, Chaparala S, et al. Mechanical shock testing and modeling of $\mathrm{PC}$ motherboards $[\mathrm{C}] / /$ Electronic Components and Technology Conference, 2004. Proceedings. IEEE, 2004:1047-1054 Vol.1.

[12] Zhang B, Ding H, Sheng X J. Modal analysis of board-level electronic package[J]. Microelectronic Engineering, 2008, 85(3):610-620. 